\title{
A POSTERIORI ERROR ESTIMATES FOR BOUNDARY ELEMENT METHODS
}

\author{
CARSTEN CARSTENSEN AND ERNST P. STEPHAN
}

\begin{abstract}
This paper deals with a general framework for a posteriori error estimates in boundary element methods which is specified for three examples, namely Symm's integral equation, an integral equation with a hypersingular operator, and a boundary integral equation for a transmission problem. Based on these estimates, an analog of Eriksson and Johnson's adaptive finite element method is proposed for the $h$-version of the Galerkin boundary element method for integral equations of the first kind. The efficiency of the approach is shown by numerical experiments which yield almost optimal convergence rates even in the presence of singularities.
\end{abstract}

\section{INTRODUCTION}

The construction of an adaptive mesh refinement procedure is of very high practical importance in the numerical analysis of partial differential equations, and we refer to the pioneering work of Babuška and Miller [3] and Eriksson and Johnson $[10,11]$. Whereas the main features of adaptivity for finite element methods now seem to be visible, and the door is open to implementation [15], comparably little is known for boundary element methods for integral equations (see e.g. $[1,13,18,19,24])$.

In this paper a new adaptive $h$-version of the Galerkin discretization for the boundary element method is presented based on a posteriori error estimates. A general framework for these a posteriori error estimates is derived in $\S 2$, and three examples are discussed in $\S \S 3-5$ involving the Dirichlet problem, the Neumann problem (for a closed and an open surface), and a transmission problem for the Laplacian, leading to integral equations with strongly elliptic pseudodifferential operators.

Even for smooth data the lack of regularity of the solution near corners (of a polygonal domain $\Omega$ ) leads to poor solutions of the numerical schemes unless appropriate singular functions are incorporated in the trial space or a suitable mesh refinement is used. In practical problems such information is missing, e.g., when we have singular (or nearly singular) data and the main problem is how to balance a graded mesh refinement towards singularities and a global

Received by the editor August 23, 1993 and, in revised form, March 2, 1994.

1991 Mathematics Subject Classification. 65N35, 65R20, 65D07, 45L10.

Key words and phrases. A posteriori error estimates, boundary element methods, adaptive boundary element methods.

The work was partly supported by DFG research group at the University of Hannover. 
almost quasioptimal refinement. The convergence results in the literature are in general asymptotic, i.e., a proposed refinement leads to a certain order of convergence which can be expected if the mesh is very fine, but it is not obvious how to handle only a few elements in order to get some optimal meshes. Hence, an adaptive feedback procedure is desired where the algorithm itself decides when and where to refine the mesh in order to improve the computed Galerkin solution. One such strategy is proposed in $\S 6$ and is based on a posteriori estimates following ideas of Johnson and Eriksson (proposed for the finite element method). Although we are far from proving efficiency of the general algorithm, our numerical experiments in $\S 7$ yield almost optimal convergence results and confirm that the algorithm gives good triangulations.

In contrast to the a posteriori error estimates in $[21,24,25]$ which are restricted to closed smooth curves in two dimensions, our general framework covers also open arcs, corners and problems in any dimension. The results in $[21,24,25]$ give-in some sense-a local a posteriori error estimate, whereas our approach leads to an upper bound of the global error in energy norms consisting of terms which can be evaluated locally. Finally, here we need no restriction at all on the mesh for two-dimensional problems (contrary to $[8,9]$ ) whereas the analysis in $[21,25]$ is performed for K-meshes only (i.e., the quotient of the element lengths of two neighboring one-dimensional elements is uniformly bounded).

We remark that the general framework in $\S 2$ is not restricted to the three examples discussed here. The ideas can easily be adapted to other problems, e.g., crack and wave problems, problems in thermodynamics, elasticity, and magnetostatics.

As shown by numerical examples, the new adaptive algorithm is efficient. However, a mathematical proof of efficiency remains an important open problem.

\section{ABSTRACT FRAMEWORK OF A POSTERIORI ERROR BOUNDS FOR BOUNDARY INTEGRAL EQUATIONS}

In finite element methods, adaptive feedback algorithms are often based on the residual. The adapted analog for boundary integral equations presented in this paper can be generally described as follows and is specified for concrete situations in the subsequent sections.

Let $X, Y$ be Banach spaces and let the operator

$$
A: X \rightarrow Y \text { be linear, bounded, and bijective . }
$$

Given some right-hand side $f \in Y$, let $u:=A^{-1} f \in X$ be the continuous solution of the equation

$$
A u=f,
$$

and let $u_{h}$ be some approximation of $u$. Define the residual $R$ by

$$
R:=f-A u_{h},
$$

and assume that the residual can be computed, at least numerically. By the inverse mapping theorem, $A^{-1}$ is bounded with a bound $\left\|A^{-1}\right\|_{L(Y ; X)}$ say, 
such that

$$
\left\|u-u_{h}\right\|_{X}=\left\|A^{-1} R\right\|_{X} \leq\left\|A^{-1}\right\|_{L(Y ; X)} \cdot\|R\|_{Y} .
$$

For motivation, let $u_{h}$ be obtained by some Galerkin procedure so that we have more information on $u_{h}$ and $R$. Thus, there exists a finite-dimensional subspace $T_{h}$ of $Y^{*}$, the (algebraic and topological) dual of $Y$, such that

$$
0=\left\langle t_{h}, R\right\rangle_{Y^{*} \times Y} \quad \text { for all } t_{h} \in T_{h},
$$

where $\langle,\rangle_{Y^{*} \times Y}$ denotes the dual pairing between $Y^{*}$ and $Y$.

Lemma 1. There exists some $\rho \in Y^{*}$ with $\|\rho\|_{Y^{*}}^{2}=\|R\|_{Y}^{2}=\langle\rho, R\rangle_{Y^{*} \times Y}$.

Proof. The lemma follows from the Hahn-Banach theorem, compare e.g. [20, Theorem 3.3], which yields existence of some functional $\tilde{\rho} \in Y^{*}$ with norm 1 and $\|R\|_{Y}=\langle\tilde{\rho}, R\rangle_{Y^{*} \times Y}$. Then, $\rho=\|R\|_{Y} \cdot \tilde{\rho}$ satisfies the assertion.

With some $\rho$ chosen as in the lemma, (2.2) shows that

$$
\begin{aligned}
\|R\|_{Y}^{2} & =\langle\rho, R\rangle_{Y^{*} \times Y}=\inf _{t_{h} \in T_{h}}\left\langle\rho-t_{h}, R\right\rangle_{Y^{*} \times Y} \\
& \leq\|R\|_{Y} \cdot \inf _{t_{h} \in T_{h}}\left\|\rho-t_{h}\right\|_{Y^{*}}
\end{aligned}
$$

whence

$$
\|R\|_{Y} \leq \inf _{t_{h} \in T_{h}}\left\|\rho-t_{h}\right\|_{Y^{*}}
$$

Comparing this with (2.1) yields the abstract a posteriori error estimate

$$
\left\|u-u_{h}\right\|_{X} \leq\left\|A^{-1}\right\|_{L(Y ; X)} \cdot \inf _{t_{h} \in T_{h}}\left\|\rho-t_{h}\right\|_{Y^{*}}
$$

When dealing with boundary integral equations, one of the typical problems arises from the fact that the "natural" spaces are often Sobolev spaces with noninteger order, which are defined, e.g., via interpolation of Banach spaces [2]. Combining the interpolation arguments with the above abstract a posteriori error estimate, we prove the following more explicit estimate, which is illustrated in this paper for three examples.

Theorem 1. Let $X, Y_{0}, Y_{\theta}, Y_{1}$ be Banach spaces with $Y_{1} \subseteq Y_{\theta} \subseteq Y_{0}$. Let $c_{\theta}$ be some positive constant with

$$
\|y\|_{Y_{\theta}} \leq c_{\theta} \cdot\|y\|_{Y_{0}}^{1-\theta} \cdot\|y\|_{Y_{1}}^{\theta} \quad \text { for all } y \in Y_{1} .
$$

Let $A: X \rightarrow Y_{\theta}$ be linear, bounded, and bijective. Let $f \in Y_{\theta}, u:=A^{-1} f$, and let $u_{h} \in X$ with $R:=f-A u_{h} \in Y_{1}$ satisfy

$$
0=\left\langle t_{h}, R\right\rangle_{Y_{0}^{*} \times Y_{0}} \quad \text { for all } t_{h} \in T_{h},
$$

where $\langle,\rangle_{Y_{0}^{*} \times Y_{0}}$ denotes the dual pairing between $Y_{0}^{*}$ and $Y_{0}$, and $T_{h}$ is a subspace of $Y_{0}^{*}$. Let $\rho \in Y_{0}^{*}$ be defined as in Lemma 1 (with $Y_{0}$ replacing $Y$ ), 
i.e., with

$$
\|\rho\|_{Y_{0}^{*}}^{2}=\|R\|_{Y_{0}}^{2}=\langle\rho, R\rangle_{Y_{0}^{*} \times Y_{0}}
$$

Then we have

$$
\left\|u-u_{h}\right\|_{X} \leq c_{\theta} \cdot\left\|A^{-1}\right\|_{L\left(Y_{\theta} ; X\right)} \cdot\|R\|_{Y_{1}}^{\theta} \cdot \inf _{t_{h} \in T_{h}}\left\|\rho-t_{h}\right\|_{Y_{0}^{*}}^{1-\theta} .
$$

Proof. Using (2.1) with $Y_{\theta}$ replacing $Y$, and estimating $\|R\|_{Y_{\theta}}$ via (2.4), we obtain

$$
\left\|u-u_{h}\right\|_{X} \leq c_{\theta} \cdot\left\|A^{-1}\right\|_{L\left(Y_{\theta} ; X\right)} \cdot\|R\|_{Y_{1}}^{\theta} \cdot\|R\|_{Y_{0}}^{1-\theta} .
$$

By Lemma 1 we have some $\rho$ satisfying (2.6). Using (2.3) with $Y_{0}$ replacing $Y$ leads to a bound of $\|R\|_{Y_{0}}$, which concludes the proof.

Theorem 1 is applied to Symm's integral equation, an integral equation with a hypersingular operator, and a boundary integral equation for a transmission problem in the following sections. In particular, we can explicitly determine $\rho$ in these examples.

Remark 1. In applications we have that the norms in $Y_{0}$ and $Y_{1}$ are "local" (like $L^{2}(\Gamma)$ or $H^{1}(\Gamma)$ ), i.e., they can be computed for any element of a triangulation (in a boundary element method for instance), and then they are given as a certain sum over all elements (compare e.g. (6.1) in §6).

Remark 2. Although Theorem 1 is simply based on the interpolation estimate (2.4) and some duality arguments, the main point is that in boundary element methods we do have orthogonality $(2.2)$ in the $L^{2}$-sense which generalizes to $H^{s}(\Gamma) \times H^{-s}(\Gamma)$ for all $s$ with $-1 \leq s \leq 1$ if $\Gamma$ is a closed Lipschitz curve. This is why we may apply (2.2) in those duality pairings-contrary to the situation in finite element methods, where (2.2) holds e.g. in the $H^{1}(\Omega)$ inner product.

Remark 3. Note that we use only that $A$ is a bijective mapping between the energy space and its dual, i.e., we need no regularity assumption on the solution, which is in general not available (cf. $\S 5$ and Remark 9).

\section{SYMM'S INTEGRAL EQUATION}

Given a bounded Lipschitz domain $\Omega \subseteq \mathbb{R}^{n}$ with boundary $\Gamma$, the Dirichlet problem for the Laplacian is related to the so-called Symm's integral equation for the unknown density $\phi$ on $\Gamma$,

$$
V \phi(x)=g(x) \quad(x \in \Gamma)
$$

with the weakly singular operator $V$ of the single-layer potential,

$$
V \phi(x):=\int_{\Gamma} \phi(y) G(x, y) d s_{y} .
$$

Here $G(x, y):=-\frac{1}{\pi} \log |x-y|$ for $n=2, G(x, y):=c_{n} \cdot|x-y|^{-n}$ for $n \geq 3$ with some constant $c_{n}>0$. In (3.1) the right-hand side $g$ is some function determined by the given Dirichlet data $\left.u\right|_{\Gamma}$. 
Let $H^{s}(\Gamma), 0 \leq s \leq 1$, be the usual Sobolev space on $\Gamma, H^{0}(\Gamma)=L^{2}(\Gamma)$, and $H^{-s}(\Gamma)$ the dual space of $H^{s}(\Gamma)$ with respect to the duality $\langle$,$\rangle which is$ defined for smooth functions $u, v$ by

$$
\langle u, v\rangle=\int_{\Gamma} u \cdot v d s .
$$

The operator $V$ is a Fredholm operator of index zero, it is injective in $L^{1}(\Gamma)$ for $n \geq 3$ or for $n=2$ if $\operatorname{cap}(\Gamma) \neq 1$ [22]. Thus, $V$ is bijective from $H^{s-1}(\Gamma)$ onto $H^{s}(\Gamma)$ in these cases. It is therefore assumed throughout this paper that in the two-dimensional case we have $1 \neq \operatorname{cap}(\Gamma)$, where $\operatorname{cap}(\Gamma)$ denotes the capacity, or conformal radius, or transfinite diameter of $\Gamma$ (cf. e.g. $[4,6,22]$ for proofs).

Let $T_{h}:=S_{h}^{0} \subseteq L^{2}(\Gamma)$ denote the vector space of piecewise polynomials with respect to a "triangulation" of the boundary

$$
\Gamma=\bigcup_{j=1}^{N} \Gamma_{j},
$$

where the "elements" $\Gamma_{1}, \ldots, \Gamma_{N}$ are either equal or have at most one common point or side, respectively. We assume that $\Gamma_{j}$ is an interval for $n=2$ or a triangle for $n=3$ etc. Define

$$
h: \Gamma \rightarrow(0, \infty), x \mapsto \operatorname{diam}\left(\Gamma_{j}\right) \text { for } x \in \Gamma_{j},
$$

where $\operatorname{diam}\left(\Gamma_{j}\right)>0$ denotes the element size.

Let $\phi_{h} \in T_{h}$ denote the Galerkin solution of (3.1), i.e.,

$$
0=\left\langle t_{h}, R\right\rangle \quad \text { for all } t_{h} \in T_{h},
$$

where $R:=g-V \phi_{h}$. Assume that $g \in L^{2}(\Gamma)$, so that, according to $T_{h} \subseteq$ $L^{2}(\Gamma)$, we have $R=V\left(\phi-\phi_{h}\right) \in H^{1}(\Gamma)$, owing to the mapping properties of the single-layer potential [4]. Let $\nabla_{\Gamma}$ denote the gradient with respect to $\Gamma$.

Theorem 2. Under the above assumptions, we have for $0 \leq s \leq 1$

$$
\left\|\phi-\phi_{h}\right\|_{H^{-s}(\Gamma)} \leq\left\|V^{-1}\right\|_{L\left(H^{1-s}(\Gamma) ; H^{-s}(\Gamma)\right)} \cdot\|R\|_{H^{1}(\Gamma)}^{1-s} \cdot\left\|h \cdot \nabla_{\Gamma} R\right\|_{L^{2}(\Gamma)}^{s} \cdot
$$

Proof. Apply Theorem 1 with $A=V, X=H^{-s}(\Gamma), Y_{0}=L^{2}(\Gamma), Y_{1}=H^{1}(\Gamma)$, $\theta=1-s, Y_{\theta}=H^{1-s}(\Gamma)$, so that $(2.4)$ holds with $c_{\theta}=1$. Note that $\rho=R$ satisfies (2.6), and there holds

$$
\inf _{t_{h} \in T_{h}}\left\|R-t_{h}\right\|_{L^{2}(\Gamma)} \leq\left\|h \cdot \nabla_{\Gamma} R\right\|_{L^{2}(\Gamma)}
$$

by standard interpolation/approximation arguments. Hence, Theorem 1 yields the assertion.

Remark 4. Using more regularity of $R$ and higher-order polynomials in $T_{h}$, one concludes, e.g. for $n=2$, that $\left\|h \cdot \nabla_{\Gamma} R\right\|_{L^{2}(\Gamma)}=\left\|h \cdot R^{\prime}\right\|_{L^{2}(\Gamma)}$ can be replaced in Theorem 2 by $c_{p} \cdot\left\|h^{p+1} \cdot\left(\frac{\partial}{\partial s}\right)^{p+1} R\right\|_{L^{2}(\Gamma)}$, where $c_{p}$ is a constant depending on $p$. The piecewise constant function $p: \Gamma \rightarrow\{0,1,2,3, \ldots\}$ denotes locally the degrees (i.e., $\left\{\left.t_{h}\right|_{\Gamma_{j}}: t_{h} \in T_{h}\right\}$ includes all polynomials of degree $\left.\left.p\right|_{\Gamma_{j}}\right)$. 
Remark 5. In Theorem 2 the term $\|R\|_{H^{1}(\Gamma)}$ can be replaced by $c \cdot\left\|R^{\prime}\right\|_{L^{2}(\Gamma)}$, where $c>0$ is a constant. For a proof, compare Lemma 3 below and note that $\langle 1, R\rangle=0$, owing to (3.2).

\section{INTEGRAL EQUATION WITH HYPERSINGULAR OPERATOR}

In this section we treat two examples for the hypersingular integral equation, namely for a two-dimensional problem (related to a closed curve $\Gamma$ ) and for a three-dimensional problem (related to an open surface $\Gamma$ ).

Given a two-dimensional bounded domain $\Omega$ with polygonal boundary $\Gamma$, the Neumann problem for the Laplacian is related to the integral equation

$$
W v(x)=f(x) \quad(x \in \Gamma)
$$

for the unknown displacement $v$ on $\Gamma$, where $W$ is the hypersingular operator

$$
W v(x):=-\frac{1}{\pi} \frac{\partial}{\partial n_{x}} \int_{\Gamma} v(y) \frac{\partial}{\partial n_{y}} \log |x-y| d s_{y} .
$$

In order to analyze (4.1), we need the following two lemmas, where

$$
H_{0}^{s}(\Gamma):=\left\{\psi \in H^{s}(\Gamma):\langle 1, \psi\rangle=0\right\},
$$

and \langle\rangle is the duality between $H^{-1 / 2}(\Gamma)$ and $H^{1 / 2}(\Gamma)$; a prime or $\frac{\partial}{\partial s}$ denotes differentiation with respect to the arc length.

Lemma 2 ([17]). We have $\langle W v, w\rangle=\left\langle V \frac{\partial v}{\partial s}, \frac{\partial w}{\partial s}\right\rangle$ for any $v, w \in H^{1 / 2}(\Gamma)$, i.e., $W=-\frac{\partial}{\partial s} V \frac{\partial}{\partial s}$.

Each $f \in L^{2}(\Gamma)$ may be integrated along the boundary with respect to the arc length, which yields a function $F \in H^{1}(\Gamma)$ if $\langle f, 1\rangle=0$ (then $F$ is globally continuous and single-valued). So far, $F$ is defined up to a constant which is fixed by $\langle F, 1\rangle=0$. Then the mapping $I: f \mapsto F$ defines an operator $I: H_{0}^{0}(\Gamma) \rightarrow H_{0}^{1}(\Gamma)$.

Lemma 3. After changing to an equivalent norm in $H_{0}^{s}(\Gamma), s \in[0,1]$, the operator $I: H_{0}^{s-1}(\Gamma) \rightarrow H_{0}^{s}(\Gamma)$ is isometric with

$$
\frac{\partial}{\partial s}(I \phi)=\phi \quad \text { for all } \phi \in H_{0}^{s-1}(\Gamma) .
$$

In other words, differentiation is an isomorphism between $H_{0}^{s}(\Gamma)$ and $H_{0}^{s-1}(\Gamma)$ with an inverse $I$.

Proof. The mapping $I: H_{0}^{0}(\Gamma) \rightarrow H_{0}^{1}(\Gamma)$ is linear and bounded if we consider the $H^{-1}$-norm in $H_{0}^{0}(\Gamma)$ and the $L^{2}$-norm in $H_{0}^{1}(\Gamma)$. Hence we may extend $I$ uniquely to a bounded linear mapping $I: H_{0}^{-1} \rightarrow H_{0}^{0}(\Gamma)$ which is the inverse of differentiation. Thus, $I$ is isometric if we endow $H_{0}^{-1}(\Gamma)$ with the equivalent norm

$$
\|\phi\|_{H_{0}^{-1}(\Gamma)}:=\|I \phi\|_{L^{2}(\Gamma)} \quad\left(\phi \in H_{0}^{-1}(\Gamma)\right) .
$$

Therefore, the assertion for arbitrary $s, 0<s<1$, follows by interpolation [2]. 
As a direct consequence of Lemmas 2 and 3 we have, by the mapping properties of $V$, that

$$
W: H_{0}^{s}(\Gamma) \rightarrow H_{0}^{s-1}(\Gamma)
$$

is linear, bounded and bijective.

Let $T_{h}:=S_{0 h}^{1} \subseteq H_{0}^{1}(\Gamma)$ be the vector space of continuous and piecewise polynomials (with respect to a "triangulation" of the one-dimensional boundary $\Gamma$ as explained in the previous section) with integral mean zero. Given $f \in$ $H_{0}^{0}(\Gamma)$, let $v_{h} \in T_{h}$ denote the Galerkin solution, i.e.,

$$
0=\left\langle t_{h}, R\right\rangle \quad \text { for all } t_{h} \in T_{h},
$$

where $R:=f-W v_{h} \in H_{0}^{1}(\Gamma)$.

Theorem 3. Under the above assumptions, we have for $0 \leq s \leq 1$

$$
\left\|v-v_{h}\right\|_{H^{s}(\Gamma)} \leq\left\|W^{-1}\right\|_{L\left(H_{0}^{s-1}(\Gamma) ; H_{0}^{s}(\Gamma)\right)} \cdot\|R\|_{L^{2}(\Gamma)}^{s} \cdot\|h \cdot R\|_{L^{2}(\Gamma)}^{1-s} \cdot
$$

Proof. Apply Theorem 1 with $A=W, X=H_{0}^{s}(\Gamma), Y_{0}=H_{0}^{-1}(\Gamma), Y_{1}=$ $H_{0}^{0}(\Gamma), \theta=s, Y_{\theta}=H_{0}^{s-1}(\Gamma)$, so that $(2.4)$ holds with $c_{\theta}=1$. Note that, by construction of the Sobolev spaces $H^{s}(\Gamma)$, the duality in (4.2) is the duality between $L^{2}(\Gamma)$ and $L^{2}(\Gamma)$ as well as between $H_{0}^{1}(\Gamma)$ and $H_{0}^{-1}(\Gamma)$. Hence, (2.5) is satisfied. Note that integrating $-R$ twice with respect to the arc length gives $\rho$, whence $\rho=-I \circ I(R)$ satisfies (2.6). Therefore,

$$
\inf _{t_{h} \in T_{h}}\left\|\rho-t_{h}\right\|_{H_{0}^{1}(\Gamma)}=\inf _{t_{h} \in T_{h}}\left\|I R-t_{h}^{\prime}\right\|_{L^{2}(\Gamma)} \leq\|h \cdot R\|_{L^{2}(\Gamma)}
$$

by choosing $t_{h}^{\prime}$ as the $L^{2}$-projection of $I R$ and using standard interpolation/ approximation arguments. Hence, Theorem 1 yields the assertion.

Remark 6. As mentioned in Remark 4, using more regularity of $R$ and higherorder polynomials in $T_{h}$, one concludes that $\|h \cdot R\|_{L^{2}(\Gamma)}$ can be replaced in Theorem 3 by $c_{p} \cdot\left\|h^{p} \cdot\left(\frac{\partial}{\partial s}\right)^{p-1} R\right\|_{L^{2}(\Gamma)}$.

Remark 7. In the numerical realization we use the space $S_{h}^{1}$ of piecewise linear trial functions without the restriction of having mean value zero. Given $(f, b) \in L^{2}(\Gamma) \times \mathbb{R}$, the problem results (see [5]) of finding $(u, a) \in H^{1}(\Gamma) \times \mathbb{R}$ with

$$
W u+a=f \quad \text { and } \quad\langle u, 1\rangle=b,
$$

and the Galerkin scheme consists in finding $\left(u_{h}, a_{h}\right) \in S_{h}^{1} \times \mathbb{R}$ with

$$
\begin{aligned}
\left\langle W v_{h}+a_{h}, w_{h}\right\rangle & =\left\langle f, w_{h}\right\rangle \quad \text { for all } w_{h} \in S_{h}^{1}, \\
\left\langle u_{h}, 1\right\rangle & =b .
\end{aligned}
$$

Using exact arithmetic, we have $u-u_{h} \in H_{0}^{1}(\Gamma), a_{h}=\frac{\langle 1, f\rangle}{\langle 1,1\rangle}$ whence $f-a_{h} \in$ $L_{0}^{2}(\Gamma)$. Then, Theorem 3 can be applied.

The second example for a hypersingular integral equation is related to screen problems in three dimensions, namely to the Neumann problem for the Laplacian outside of an open surface. It is known (cf. e.g. [12, 23] and the references 
therein) that this problem can be equivalently rewritten as a hypersingular integral equation

$$
W v(x):=-\frac{1}{4 \pi} \frac{\partial}{\partial n_{x}} \int_{\Gamma}\left(\frac{\partial}{\partial n_{y}} \frac{1}{|x-y|}\right) v(y) d s_{y}=f(x) \quad(x \in \Gamma) .
$$

Here, the integral

$$
\frac{\partial}{\partial n_{x}} \int_{\Gamma} \frac{\partial}{\partial n_{y}} \frac{1}{|x-y|} v(y) d s_{y}=\int_{\Gamma} \frac{1}{|x-y|^{3}} v(y) d s_{y}
$$

has to be understood as a "Hadamard finite-part integral" and $v$ gives the jump across the screen $\Gamma$ of the solution of the original Neumann problem. For notational simplicity, we take $\Gamma:=[0,1]^{2} \times\{0\} \equiv[0,1]^{2}$. Then, let $\tilde{\Gamma}$ be a smooth, bounded, closed surface containing $\Gamma$. We use the following Sobolev spaces for real $s[14,16]$ :

$$
\begin{aligned}
H^{s}(\tilde{\Gamma}) & :=\left\{\left.u\right|_{\tilde{\Gamma}}: u \in H^{s+1 / 2}\left(\mathbb{R}^{3}\right)\right\} \quad(\text { for } s>0), \\
H^{0}(\tilde{\Gamma}) & :=L^{2}(\tilde{\Gamma}), \\
H^{-s}(\tilde{\Gamma}) & :=\left(H^{s}(\tilde{\Gamma})\right)^{*} \quad(\text { dual space) } \quad(\text { for } s>0), \\
H^{s}(\Gamma) & :=\left\{\left.u\right|_{\Gamma}: u \in H^{s}(\tilde{\Gamma})\right\} \quad(\text { for all } s), \\
\tilde{H}^{s}(\Gamma) & :=\left\{u \in H^{s}(\tilde{\Gamma}): \operatorname{supp} u \subseteq \bar{\Gamma}\right\} \quad(\text { for all } s) .
\end{aligned}
$$

Note that $\tilde{H}^{1 / 2}(\Gamma)=H_{00}^{1 / 2}(\Gamma)$ in [16]. It is known (cf. e.g. $[12,23]$ and the references therein) that the hypersingular operator $W$ maps $\tilde{H}^{1 / 2+s}(\Gamma)$ onto $H^{-1 / 2+s}(\Gamma)$ and is bounded, linear and bijective for all real $s$ with $|s|<1 / 2$.

Let $T_{h}:=S_{h}^{1} \subseteq \tilde{H}^{1}(\Gamma)$ be the space of continuous piecewise linear trial functions (with respect to a regular triangulation of the two-dimensional domain $\Gamma$ where the triangulation satisfies the angle condition) which vanish at the boundary $\partial \Gamma$ of $\Gamma$. Then, the Galerkin equations (cf., e.g., [12]) yield that $\left\langle R, w_{h}\right\rangle_{H^{-1 / 2}(\Gamma) \times \tilde{H}^{1 / 2}(\Gamma)}=0$ for any $w_{h} \in T_{h}$, where $R:=f-W u_{h}$.

From the general framework in $\S 2$ we obtain the following a posteriori error estimate. The local element diameter is denoted by the piecewise constant function $h \in L^{\infty}(\Gamma)$.

Theorem 4. Under the above assumptions there exists a constant $C>0$ such that for $0 \leq s<1$ and $f \in L^{2}(\Gamma)$

$$
\left\|v-v_{h}\right\|_{\tilde{H}^{s}(\Gamma)} \leq C \cdot\left\|W^{-1}\right\|_{L\left(H^{s-1}(\Gamma) ; \tilde{H}^{s}(\Gamma)\right)} \cdot\|R\|_{L^{2}(\Gamma)}^{s}\left\|h \cdot D^{2} \rho\right\|_{L^{2}(\Gamma)}^{1-s},
$$

where $D^{2}:=\left(\frac{\partial^{2}}{\partial x_{i} \partial x_{j}}: i, j=1,2\right)$ denotes the vector of all second-order partial derivatives with respect to $x_{1}$ and $x_{2}$. Here, $\rho \in \tilde{H}^{2}(\Gamma)$ is the solution of the problem

$$
\Delta \rho=-R \text { in } \Gamma, \quad \rho=0 \text { on } \partial \Gamma .
$$

Proof. Since $u_{h} \in T_{h}$ lies in $H^{1}(\tilde{\Gamma})$, we have $W u_{h} \in L^{2}(\tilde{\Gamma})$, whence $\left.\left(W u_{h}\right)\right|_{\Gamma}=$ : $W u_{h} \in L^{2}(\Gamma)$. Thus, $R:=f-W u_{h} \in L^{2}(\Gamma)$. Compare, however, Remark 9.

Therefore, we may apply Theorem 1 with $A=W, X=\tilde{H}^{s}(\Gamma), \quad Y_{0}=$ $H^{-1}(\Gamma), Y_{1}=L^{2}(\Gamma), Y_{\theta}=H^{s-1}(\Gamma), \theta=s$, so that (2.4) holds with $c_{\theta}=1$. 
In $\tilde{H}^{1}(\Gamma)$ the seminorm is an equivalent norm and we may endow $\tilde{H}^{1}(\Gamma)$ with the norm $\left\|\nabla_{\Gamma} \cdot\right\|_{L^{2}(\Gamma)}$ where $\nabla_{\Gamma}=\left(\frac{\partial}{\partial x_{1}}, \frac{\partial}{\partial x_{2}}\right)$.

Note that $(2.5)$ is satisfied, so that it remains to determine $\rho$ with $\|\rho\|_{Y_{0}^{*}}^{2}=$ $\|R\|_{Y_{0}}^{2}=\langle\rho, R\rangle_{Y_{0}^{*} \times Y_{0}}$. Consider $\rho \in H^{1}(\Gamma)$, the solution of (4.4), which, since $R \in L^{2}(\Gamma)$ and $\Gamma$ is convex, lies also in $H^{2}(\Gamma)$. For this $\rho$ we have by duality (and the equivalent norm chosen in $\tilde{H}^{1}(\Gamma)$ )

$$
\begin{aligned}
\|R\|_{H^{-1}(\Gamma)} & =\sup _{\|\eta\|_{\dot{H}^{1}(\Gamma)} \leq 1}\langle R, \eta\rangle=\sup _{\left\|\nabla_{\Gamma} \eta\right\|_{L^{2}(\Gamma)} \leq 1}\langle\Delta \rho, \eta\rangle \\
& =\sup _{\left\|\nabla_{\Gamma} \eta\right\|_{L^{2}(\Gamma)} \leq 1}\left\langle\nabla_{\Gamma} \rho, \nabla_{\Gamma} \eta\right\rangle .
\end{aligned}
$$

Hence, according to Cauchy's inequality, $\|R\|_{H^{-1}(\Gamma)} \leq\|\rho\|_{\tilde{H}^{1}(\Gamma)}$. Taking $\eta=$ $\rho /\|\rho\|_{\hat{H}^{1}(\Gamma)}$ then proves

$$
\|R\|_{H^{-1}(\Gamma)}=\|\rho\|_{\tilde{H}^{1}(\Gamma)} .
$$

Moreover (taking the optimal $\eta=\rho /\|\rho\|_{\hat{H}^{1}(\Gamma)}$ again together with (4.4)), we conclude

$$
\|R\|_{H^{-1}(\Gamma)}^{2}=\langle R, \rho\rangle .
$$

Hence, the considered $\rho$ satisfies (2.6).

Finally, since $\rho \in H^{2}(\Gamma)$, and using standard arguments in finite element approximation, we obtain

$$
\inf _{t_{h} \in T_{h}}\left\|\rho-t_{h}\right\|_{\hat{H}^{1}(\Gamma)} \leq C \cdot\left\|h \cdot D^{2} \rho\right\|_{L^{2}(\Gamma)} .
$$

Hence, Theorem 1 yields the theorem.

Remark 8. Note that for open surfaces we have in general that a solution $v \in$ $H^{1 / 2}(\Gamma)$ of (4.3) does not lie in $H^{1}(\Gamma)$, even for a smooth right-hand side $f$ (cf. $[12,23])$. This is why $s=1$ is excluded in Theorem $4:\left\|W^{-1}\right\|_{L\left(L^{2}(\Gamma) ; \tilde{H}^{1}(\Gamma)\right.}=\infty$. Nevertheless, the residual $R$ still belongs to $L^{2}(\Gamma)$.

Remark 9. Although $W$ is not bijective as a mapping between $\tilde{H}^{1}(\Gamma)$ and $L^{2}(\Gamma)$ [23], we can prove

$$
\left\|v-v_{h}\right\|_{\tilde{H}^{s}(\Gamma)} \leq C_{1}^{s} C_{0}^{1-s} \cdot\|R\|_{H^{-1}(\Gamma)}^{1-s} \cdot\|R\|_{L^{2}(\Gamma)}^{s},
$$

as shown in Theorem 4, whereas a simple interpolation between $s=0$ and $s=1$ is not allowed.

In Theorem 4 the term $\left\|h \cdot D^{2} \rho\right\|_{L^{2}(\Gamma)}$ can be evaluated only if $\rho$ is known after solving (4.4). In the remaining part of this section we estimate the righthand side by solving (4.4) approximately via a Galerkin procedure. Thus, we obtain some unique $\rho_{h} \in T_{h}$ with

$$
\int_{\Gamma} \nabla_{\Gamma} \rho_{h} \cdot \nabla_{\Gamma} t_{h} d a=\int_{\Gamma} R \cdot t_{h} d a \quad \text { for all } t_{h} \in T_{h} .
$$

In order to give a bound for $\rho-\rho_{h}$ we use results of [11], where we need assumptions on the mesh. In addition we assume that the triangles of the triangulations have an interior angle bounded below by a constant $\theta_{0}$. We also 
need some $\bar{h} \in C^{1}(\bar{\Gamma})$ which is related to the mesh diameters described by the piecewise constant function $h$ via

$$
c_{0} \bar{h} \leq h \leq \bar{h} \text { a.e. on } \Gamma \text {. }
$$

Then, we require

$$
\left|\nabla_{\Gamma} \bar{h}\right| \leq \mu \text { a.e. on } \Gamma
$$

with some constant $\mu>0$. We emphasize that $\theta_{0}, c_{0}, \mu$ are independent of the mesh.

Since $\rho_{h}$ is piecewise linear, its gradient is piecewise constant, and hence its normal derivative has a jump $\left[\nabla_{\Gamma} \rho_{h} \cdot n\right]$ across the interior sides $E$ of the elements in the triangulation. Let

$$
D_{h, 1} \rho_{h}:=\sqrt{\sum_{E} h_{E}^{2}\left|\left[\nabla_{\Gamma} \rho_{h} \cdot n\right]\right|^{2}},
$$

where the sum is with respect to all interior sides $E$ and $h_{E}$ is the length of E.

Theorem 5 ([11, Theorem 2.2]). There exists a constant $C>0$ depending only on $\theta_{0}$ and $c_{0}$ such that if $\mu>0$ is sufficiently small, then

$$
\left\|\nabla_{\Gamma}\left(\rho-\rho_{h}\right)\right\|_{L^{2}(\Gamma)} \leq C\left(\|h \cdot R\|_{L^{2}(\Gamma)}+D_{h, 1} \rho_{h}\right),
$$

where $\rho$ solves (4.4) and $\rho_{h} \in T_{h}$ is its Galerkin approximation.

Remark 10. We remark that the right-hand side in Theorem 5 can be computed (at least numerically) and is sharp in the sense that, the right-hand side is bounded from above by $C\left\|h D^{2} \rho\right\|_{L^{2}(\Gamma)}$ (see [11, Theorem 3.2]).

Taking $t_{h}=\rho_{h}$ in the proof Theorem 4, we get from Theorem 5 the following result.

Theorem 6. Under the above assumptions, we have for $0<s<1$ with some constant $C>0$, and for $\mu>0$ sufficiently small,

$$
\left\|v-v_{h}\right\|_{\tilde{H}^{s}(\Gamma)} \leq C \cdot\|R\|_{L^{2}(\Gamma)}^{s}\left(\|h \cdot R\|_{L^{2}(\Gamma)}+D_{h, 1} \rho_{h}\right)^{1-s} .
$$

Remark 11. A similar result also holds for the Dirichlet problem and a weakly singular integral operator on the open surface.

\section{INTEGRAL EQUATION FOR A TRANSMISSION PROBLEM}

In [7] the transmission problem is rewritten into a boundary integral equation

$$
H\left(\begin{array}{l}
v \\
\phi
\end{array}\right)=\frac{1}{2}(1+H)\left(\begin{array}{l}
f \\
g
\end{array}\right) \text {, }
$$

where $(f, g) \in H^{1}(\Gamma) \times L^{2}(\Gamma)$ are prescribed jumps over the polygonal boundary $\Gamma$ of the bounded domain $\Omega$ and

$$
H:=\left(\begin{array}{cc}
-K & V \\
W & K^{\prime}
\end{array}\right): H^{s}(\Gamma) \times H^{s-1}(\Gamma) \rightarrow H^{s}(\Gamma) \times H^{s-1}(\Gamma) .
$$

Here, $V$ and $W$ are the single-layer potential and the hypersingular operator, respectively, as described in the previous sections, and $K$ is the double-layer 
potential with its dual $K^{\prime}$,

$$
\begin{aligned}
(K v)(z) & :=-\frac{1}{\pi} \int_{\Gamma} v(\zeta) \frac{\partial}{\partial n_{\zeta}} \log |z-\zeta| d s_{\zeta}, \\
\left(K^{\prime} \phi\right)(z) & :=-\frac{1}{\pi} \int_{\Gamma} \phi(\zeta) \frac{\partial}{\partial n_{z}} \log |z-\zeta| d s_{\zeta} .
\end{aligned}
$$

The operator $H$ is linear, bounded and a Fredholm operator of index zero for $s \in[0,1]$ [7] and, since the conditions in [7] are satisfied (compare [9]), $H$ is bijective, so that the transmission problem as well as the integral equation (5.1) have unique solutions $(v, \phi) \in H^{1}(\Gamma) \times L^{2}(\Gamma)$.

A boundary element method for (5.1) is described and analyzed in [7] as well. For a "triangulation" of $\Gamma$ let

$$
T_{h}:=S_{h}^{0} \times S_{h}^{1} \subseteq L^{2}(\Gamma) \times H^{1}(\Gamma),
$$

where $S_{h}^{1}$ and $S_{h}^{0}$ denote the continuous piecewise linear and (discontinuous) piecewise constant functions. Let $\left(v_{h}, \phi_{h}\right) \in T_{h}$ denote the Galerkin solution, so that the residual

$$
\begin{aligned}
R & :=\frac{1}{2}(1+H)\left(\begin{array}{c}
f \\
g
\end{array}\right)-H\left(\begin{array}{c}
v_{h} \\
\phi_{h}
\end{array}\right)=:\left(R_{1}, R_{2}\right) \in H^{1}(\Gamma) \times L^{2}(\Gamma), \\
R_{1} & :=\frac{1}{2}(f-K f+V g)-V \phi_{h}+K v_{h}, \\
R_{2} & :=\frac{1}{2}\left(g+K^{\prime} g+W f\right)-W v_{h}-K^{\prime} \phi_{h}
\end{aligned}
$$

satisfies

$$
0=\left\langle R,\left(\psi_{h}, w_{h}\right)\right\rangle=\left\langle R_{1}, \psi_{h}\right\rangle+\left\langle R_{2}, w_{h}\right\rangle \text { for all }\left(\psi_{h}, w_{h}\right) \in T_{h} .
$$

Theorem 7. Under the above assumptions, we have for $0 \leq s \leq 1$

$$
\begin{aligned}
\left\|\left(\begin{array}{l}
v \\
\phi
\end{array}\right)-\left(\begin{array}{c}
v_{h} \\
\phi_{h}
\end{array}\right)\right\|_{H^{s}(\Gamma) \times H^{s-1}(\Gamma) \leq} & \left\|H^{-1}\right\|_{L\left(H^{s}(\Gamma) \times H^{s-1}(\Gamma) ; H^{s}(\Gamma) \times H^{s-1}(\Gamma)\right)} \\
& \cdot\|R\|_{H^{1}(\Gamma) \times L^{2}(\Gamma)}^{s-1} \cdot\left\|h \cdot\left(\begin{array}{c}
R_{1}^{\prime} \\
R_{2}
\end{array}\right)\right\|_{L^{2}(\Gamma)^{2}}^{s}
\end{aligned}
$$

Proof. Apply Theorem 1 with $A=H, X=H^{s}(\Gamma) \times H^{s-1}(\Gamma), Y_{0}=L^{2}(\Gamma) \times$ $H^{-1}(\Gamma), Y_{1}=H^{1}(\Gamma) \times L^{2}(\Gamma), \theta=s, Y_{\theta}=H^{s}(\Gamma) \times H^{s-1}(\Gamma)$, so that $(2.4)$ holds with $c_{\theta}=1$. Note that $\rho=\left(R_{1},-I \circ I R_{2}\right)$ satisfies the conditions of Lemma 1 , and

$$
\inf _{t_{h} \in T_{h}}\left\|R-t_{h}\right\|_{L^{2}(\Gamma) \times H^{1}(\Gamma)} \leq\left\|h \cdot\left(\begin{array}{c}
R_{1}^{\prime} \\
R_{2}
\end{array}\right)\right\|_{L^{2}(\Gamma)^{2}}
$$

by standard interpolation/approximation arguments. Hence, Theorem 1 yields the theorem.

Remark 12. As in the previous sections, assuming more regularity of the residuals and higher degrees of the piecewise polynomials in $T_{h}$, we obtain terms with higher powers of $h$.

Remark 13. Using (5.2) and Lemma 3, one may replace $\left\|R_{1}\right\|_{H^{1}(\Gamma)}$ in Theorem 7 by $c \cdot\left\|R_{1}^{\prime}\right\|_{L^{2}(\Gamma)}$ with a constant $c$. 


\section{ADAPTIVE FEEDBACK ALGORITHM}

For a given "triangulation" of the boundary $\Gamma=\bigcup_{j=1}^{N} \Gamma_{j}$ we can compute an approximation of the contribution $a_{j}$ of one element $\Gamma_{j}$ by numerical integration. For Symm's integral equation, $a_{j}:=\left\|R^{\prime}\right\|_{L^{2}\left(\Gamma_{j}\right)}$, for the integral equation with the hypersingular operator, $a_{j}:=\|R\|_{L^{2}\left(\Gamma_{j}\right)}$, and in the case of the integral equation for the transmission problem, $a_{j}:=\left\|R_{1}^{\prime}\right\|_{L^{2}\left(\Gamma_{j}\right)}+\left\|R_{2}\right\|_{L^{2}\left(\Gamma_{j}\right)}$. Then, with some constant $c>0$, we have in all three examples that the error in the energy norm $(s=1 / 2)$ is bounded by

$$
c \cdot\left(\sum_{j=1}^{N} a_{j}^{2}\right)^{1 / 4} \cdot\left(\sum_{j=1}^{N} h_{j}^{2} \cdot a_{j}^{2}\right)^{1 / 4},
$$

where $h_{j}:=\left|\Gamma_{j}\right|$ is the length of the element $\Gamma_{j}$.

This a posteriori error estimate is not very useful for absolute error control unless the constant $c>0$ (or at least an upper bound) is known. But it can be used to compare the contributions to the local error.

Hence, we may control the process of mesh refining such that (6.1) becomes small and the error is uniformly distributed. Let $a:=\sqrt{\sum_{j=1}^{N} a_{j}^{2}}$; then the contribution of any element $\Gamma_{j}$ to the error is $c^{4} \cdot h_{j}^{2} \cdot a_{j}^{2} \cdot a^{2}$. Thus, we are led to consider $h_{j} \cdot a_{j}$ and to organize the mesh refinement so that all terms $h_{1} \cdot a_{1}, \ldots, h_{N} \cdot a_{N}$ become of the same magnitude.

The meshes in our numerical examples are controlled by the following algorithm, where $0 \leq \theta \leq 1$ is a global parameter:

Algorithm (A). Given some coarse, e.g. uniform, mesh, refine it successively by halving some of the elements, using the following rule. For any triangulation define $a_{1}, \ldots, a_{N}$ as above and refine the element $\Gamma_{j}$ if and only if

$$
h_{j} \cdot a_{j} \geq \theta \cdot \max _{k=1, \ldots, N} h_{k} \cdot a_{k} .
$$

Note that $\theta=0$ gives a uniform triangulation, whereas the number of refined elements decreases with increasing $\theta$.

Although pseudodifferential operators are nonlocal, we hope that a refinement of $\Gamma_{j}$ leads to improved bounds (6.1) for the error in the energy norm.

\section{NUMERICAL EXPERIMENTS}

We consider three numerical examples, namely the $h$-version of the Galerkin method for the weakly singular integral equation, for the hypersingular integral equation, and for the transmission problem.

7.1. Remarks on the implementation. In all cases we take continuous piecewise linear and piecewise constant trial functions $S_{h}^{1}$ and $S_{h}^{0}$, respectively.

As it is shown in $[13,18]$, the single-layer potential and the adjoint of the double-layer potential can be evaluated explicitly for piecewise polynomials. Then, the outer integration in the Galerkin scheme is performed via a Gaussian quadrature rule. Any other term is computed by applying Gaussian quadrature twice. We refer the reader to $[8,9]$ for a description of the numerical details. 

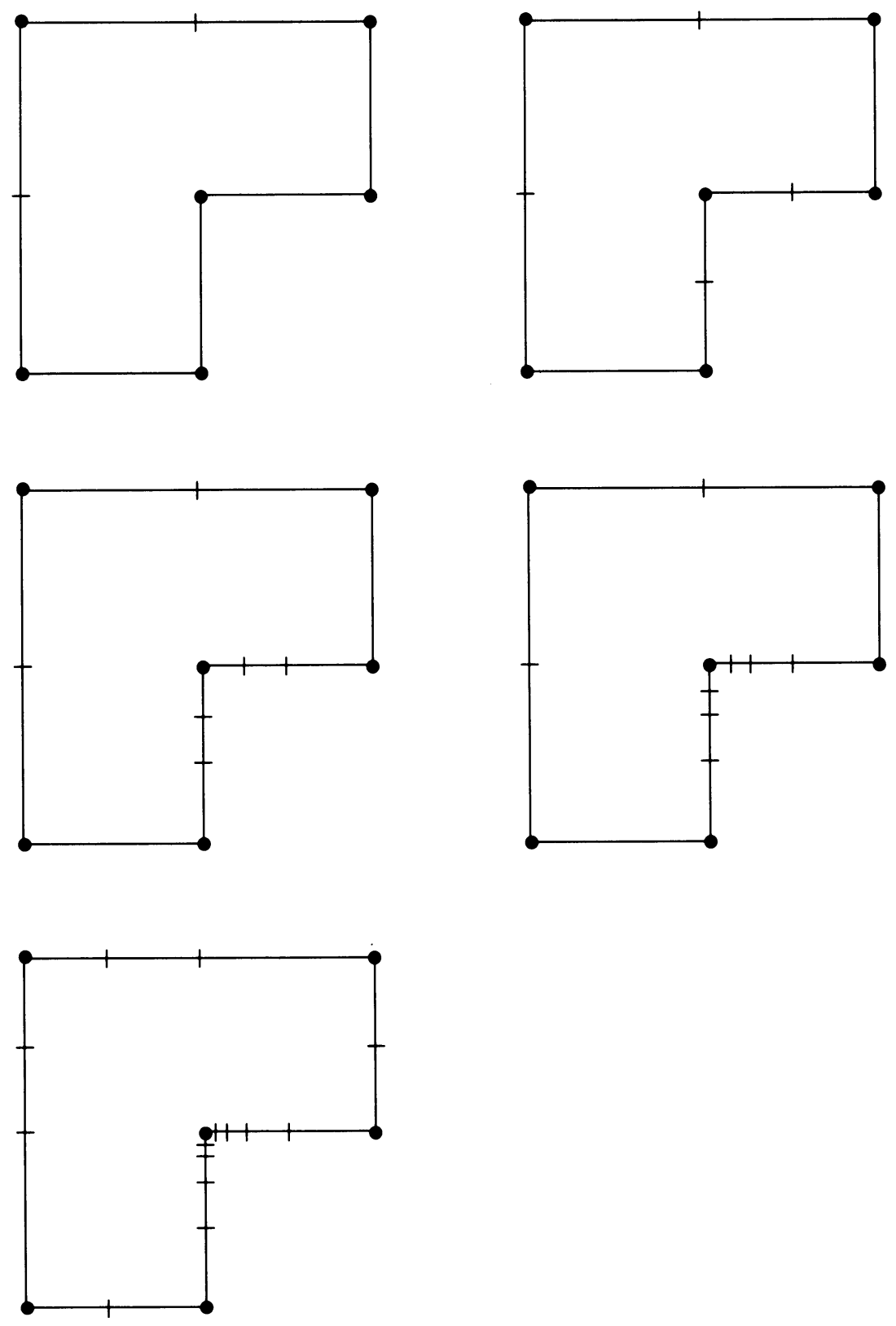

Figure 1. The meshes produced by Algorithm (A) for $\theta=0.75$ in Example 1

For $\Gamma$ we take the $L$-shaped polygon with vertices $(0,0),(1,0),(1,1)$, $(-1,1),(-1,-1),(0,-1)$, cf. Figure 1 .

We identify $\mathbb{R}^{2}$ with the complex plane and write $z=x_{1}+i x_{2}$ for a complex number, with $\Re z=x_{1}$ and $\Im z=x_{2}$.

7.2. Example 1: Symm's integral equation. On the L-shaped polygon $\Gamma$ we consider Symm's integral equation (3.1) with the solution $\phi=\Im z^{2 / 3}$. 


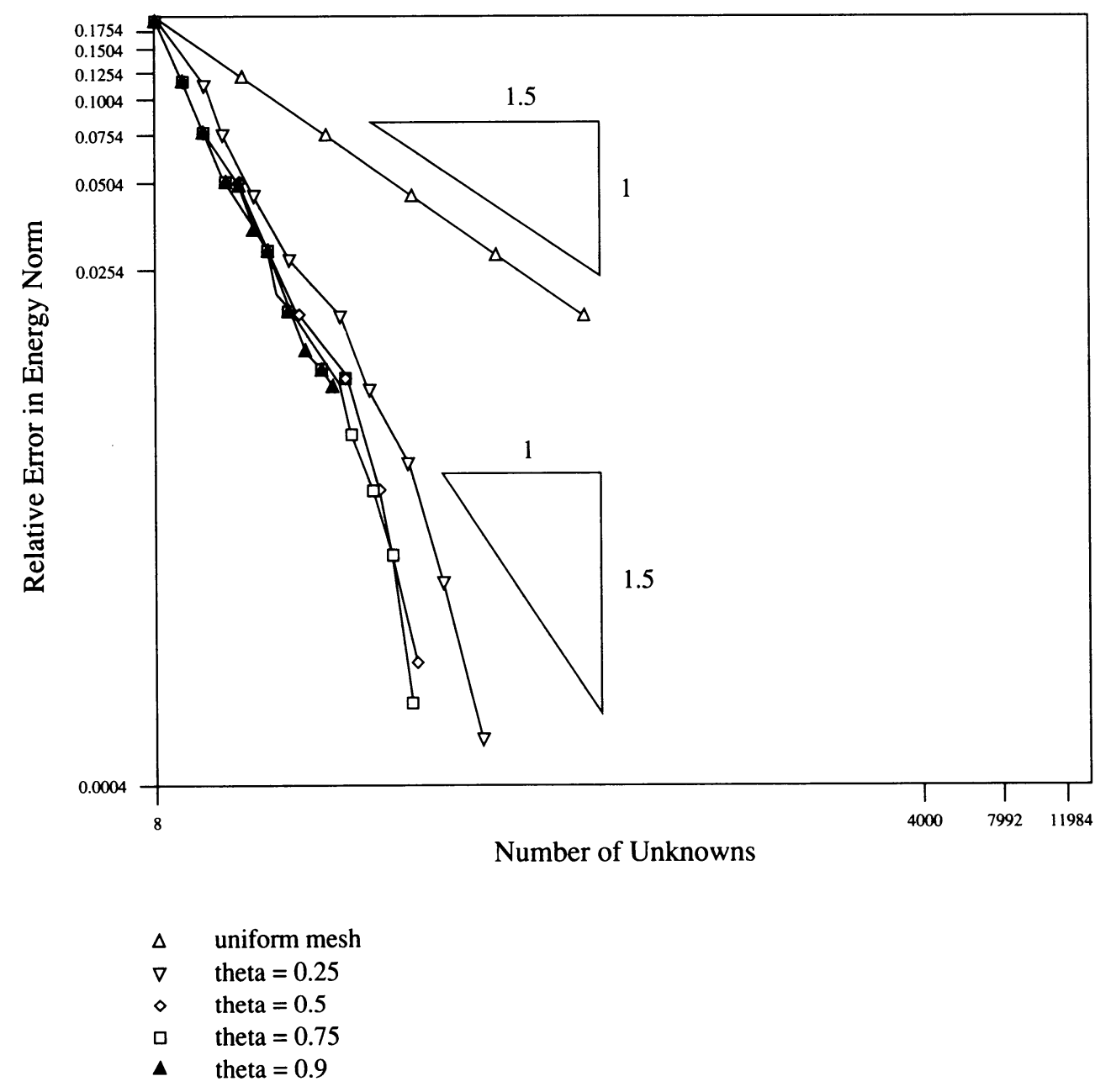

FIGURE 2. The error of the Galerkin solutions with respect to meshes produced by Algorithm (A) in Example 1

We refer to [8] for a detailed description of the numerical computations concerning the Galerkin procedure, which is an $h$-method with piecewise constant trial functions.

The given coarse mesh is shown in Figure 1 as well as the meshes produced by Algorithm (A) for $\theta=0.75$. The various convergence rates are shown in Figure 2 for $\theta=0,0.25,0.5,0.75,0.9$. Here and in the following we compute the error in the energy norm and plot the relative error versus the corresponding number of unknowns both in a logarithmic scaling.

As is well known, the convergence rates of uniform meshes, i.e., for $\theta=0$, are nonoptimal, owing to the singular behavior of the solution. In the example we expect an order of convergence $2 / 3$ for the uniform mesh $(\theta=0)$ and $3 / 2$ as the optimal value. This rate together with a considerable improvement of the produced meshes, e.g., for $\theta=0.75$, is shown in Figure 2 . Thus, our adaptive algorithm (A) is efficient and therefore our a posteriori error estimate is sharp. 
7.3. Example 2: A hypersingular integral equation. On the L-shaped polygon $\Gamma$ we consider the hypersingular integral equation (4.1) with the solution $v=$ $\Im z^{1 / 7}$. We refer to [8] for a detailed description of the numerical computations concerning the Galerkin procedure, which is an $h$-method with continuous piecewise linear trial functions (compare Remark 7). Again, the convergence rates for uniform meshes $(\theta=0)$ are nonoptimal owing to the singular behavior of the solution. This and some improvement of the produced meshes can be seen in Figure 3 where the convergence behavior is shown for $\theta=0,0.25$, $0.5,0.75,0.9$.

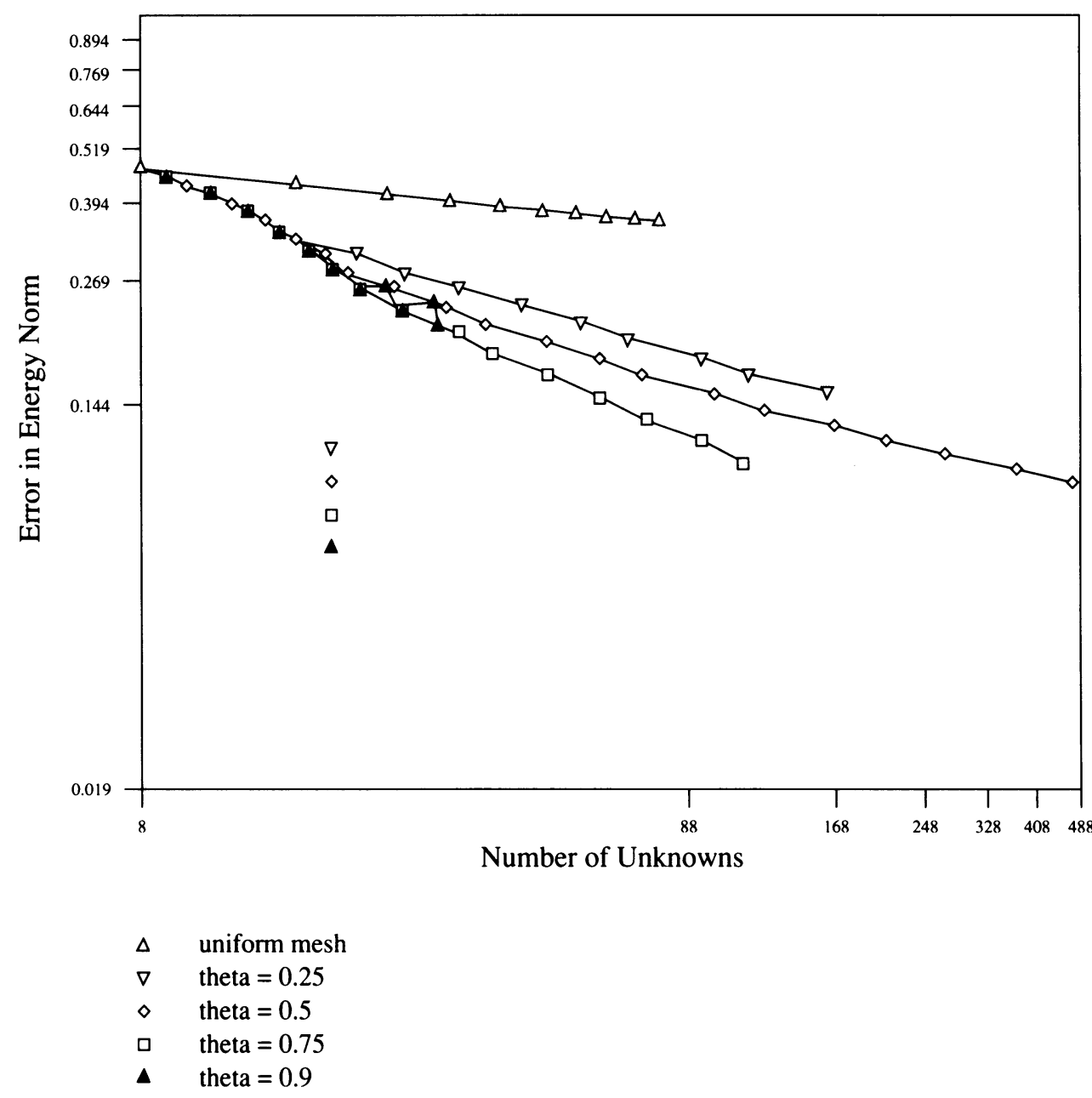

FIGURE 3. The error of the Galerkin solutions with respect to meshes produced by Algorithm (A) in Example 2 
7.4. Example 3: Transmission problem. We consider the integral equation (5.1). As an example we take the transmission problem with the solution $u_{1}=\Im z^{2 / 3}$ in $\Omega_{1}$, the $\mathrm{L}$-shaped domain, and $u_{2}=\log \left|z-\frac{1}{2}-\frac{1}{2} i\right|$ in $\Omega_{2}:=\mathbb{R}^{2} \backslash \Omega_{1}$. We refer the reader to [9] for a description of the numerical details. The resulting convergence behavior is shown in Figure 4 for $\theta=0,0.3,0.5,0.6,0.7,0.8$, 0.9 . Owing to the singularity of the solution, we get a convergence rate $2 / 3$ for a uniform refinement $(\theta=0)$ and almost an improvement to the optimal convergence rate 1.5 for $\theta$ close to 1 .

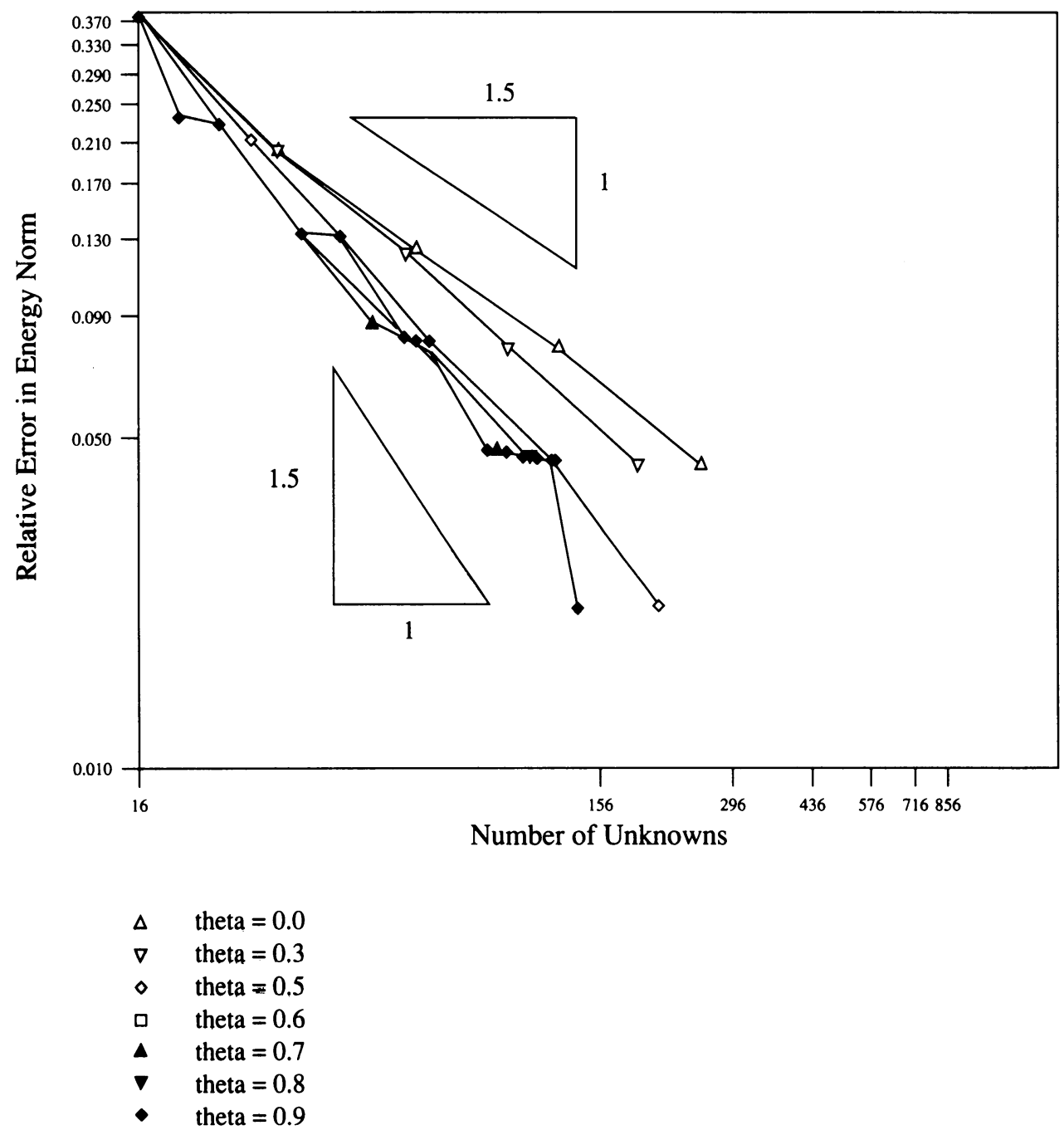

Figure 4. The error of the Galerkin solutions with respect to meshes produced by Algorithm (A) in Example 3 


\section{ACKNOWLEDGMENT}

The authors would like to thank S. Eicke and S. Zaprianov for calculating the numerical examples and the DFG Forschergruppe at the University of Hannover for support.

\section{BIBLIOGRAPHY}

1. M. Asadzadeh and K. Eriksson, An adaptive finite element method for a potential problem, Preprint.

2. J. Bergh and J. Löfström, Interpolation spaces, Springer, Berlin, 1976.

3. I. Babuška and A. Miller, A posteriori error estimates and adaptive techniques for the finite element method, Tech. Note BN-968, Institute for Physical Science and Technology, Univ. of Maryland, College Park, MD, 1981.

4. M. Costabel, Boundary integral operators on Lipschitz domains: Elementary results, SIAM J. Math. Anal. 19 (1988), 613-626.

5. M. Costabel and E.P. Stephan, The normal derivative of the double layer potential on polygons and Galerkin approximation, Appl. Anal. 16 (1983), 205-228.

6. - Boundary integral equations for mixed boundary value problems in polygonal domains and Galerkin approximation, Banach Center Publ. 15 (1985), 175-251.

7. Appl. 106 (1985), 367-413.

8. C. Carstensen and E.P. Stephan, Adaptive boundary element methods for some first kind integral equations, SIAM J. Numer. Anal. (to appear).

9. __ Adaptive boundary element methods for transmission problems, Preprint, 1993.

10. K. Eriksson and C. Johnson, An adaptive finite element method for linear elliptic problems, Math. Comp. 50 (1988), 361-383.

11. Adaptive finite element methods for parabolic problems I. A linear model problem, SIAM J. Numer. Anal. 28 (1991), 43-77.

12. V. Ervin and E.P. Stephan, A boundary element Galerkin method for a hypersingular integral equation on open surfaces, Math. Methods Appl. Sci. 13 (1990), 281-289.

13. V. Ervin, N. Heuer, and E.P. Stephan, On the $h-p$ version of the boundary element method for Symm's integral equation on polygons, Comput. Methods Appl. Mech. Engrg. 110 (1993), 25-38.

14. L. Hörmander, Linear partial differential operators., Springer-Verlag, Berlin-HeidelbergNew York, 1963.

15. C. Johnson and P. Hansbo, Adaptive finite element methods in computational mechanics, Comput. Methods Appl. Mech. Engrg. 101 (1992), 143-181.

16. J.L. Lions and E. Magenes, Non-homogeneous boundary value problems and applications, Vol. I, Springer-Verlag, Berlin-Heidelberg-New York, 1972.

17. J.C. Nedelec, La méthode des éléments finis appliquée aux équations intégrales de la physique, First meeting AFCET-SMF on applied mathematics Palaiseau, Vol. 1, 1978, pp. 181-190.

18. F.V. Postell and E.P. Stephan, On the $h$-, $p$ - and $h-p$ versions of the boundary element method-numerical results, Comput. Methods Appl. Mech. Engrg. 83 (1990), 69-89.

19. E. Rank, Adaptive boundary element methods, Boundary Elements 9 , Vol. 1 (C.A. Brebbia, W.L. Wendland, and G. Kuhn, eds.), Springer-Verlag, Heidelberg, 1987, pp. 259-273.

20. W. Rudin, Functional analysis, TATA MacGraw-Hill, 1973.

21. J. Saranen and W.L. Wendland, Local residual-type error estimates for adaptive boundary element methods on closed curves, Applicable Anal. 48 (1993), 37-50.

22. I.H. Sloan and A. Spence, The Galerkin method for integral equations of the first kind with logarithmic kernel: Theory, IMA J. Numer. Anal. 8 (1988), 105-122. 
23. E.P. Stephan, Boundary integral equations for screen problems in $\mathbb{R}^{3}$, Integral Equations Operator Theory 10 (1987), 236-257.

24. W.L. Wendland and De-hao Yu, Adaptive boundary element methods for strongly elliptic integral equations, Numer. Math. 53 (1988), 539-558.

25. __ A posteriori local error estimates of boundary element methods with some pseudodifferential equations on closed curves, J. Comput. Math. 10 (1992), 273-289.

Department of Mathematics, Heriot-Watt University, Edinburgh EH14 4AS, United KINGDOM

E-mail address: carsten€cara.ma.hw.ac.uk

INSTitut Für ANGewandte Mathematik, UNI HaNnover, Welfengarten 1, D-30167 HaNNOVER, GERMANY

E-mail address: stephaneserver.ifam.uni-hannover.de 